\title{
Effect of isoenergetic low- and high-carbohydrate diets on substrate kinetics and oxidation in healthy men
}

\author{
Christina Koutsari ${ }^{1}$ and Labros S. Sidossis ${ }^{1,2 *}$ \\ ${ }^{1}$ Laboratory of Nutrition and Clinical Dietetics, Harokopio University, E. Venizelou 70, 176 71, Athens, Greece \\ ${ }^{2}$ Department of Surgery, The University of Texas Medical Branch at Galveston, TX, USA
}

(Received 30 September 2002 - Revised 27 January 2003 - Accepted 22 March 2003)

\begin{abstract}
The effect of diet composition on post-absorptive ( $15 \mathrm{~h}$ fast) fatty acid and glucose metabolism was investigated in five healthy men after 2 weeks on a low-carbohydrate (Low-CHO) diet (30\% energy intake from carbohydrates, $55 \%$ from fat, $15 \%$ from protein) and after 2 weeks on a high-carbohydrate (High-CHO) diet (energy intake 75, 10 and $15 \%$ from carbohydrates, fat and protein respectively). The diets were isoenergetic and comprised real foods. Stable-isotope tracer methodology and indirect calorimetry were employed to measure glucose and fatty acid kinetics and oxidation. The relative contribution of carbohydrate to the total energy expenditure was significantly higher after the High-CHO diet. After the High-CHO diet, total and plasma fatty oxidation (2.4 (SE 0.7$)$ and 2.1 (SE 0.4$) \mu \mathrm{mol} / \mathrm{kg}$ per min respectively) were significantly lower than after the Low-CHO diet (4.8 (SE 0.5) and 4.6 (SE 0.8) $\mu \mathrm{mol} / \mathrm{kg}$ per min for total and plasma fatty oxidation respectively). The rate of appearance (Ra) of non-esterified fatty acids (NEFA) in plasma and the arterial NEFA concentration were both significantly lower following the High-CHO than the Low-CHO diet. However, even after the High-CHO diet, NEFA Ra was threefold higher than plasma fatty acid oxidation. Thus, the decrease in fatty acid oxidation after consumption of a high-carbohydrate diet for 2 weeks in healthy men is unlikely to result from decreased fatty acid delivery to the tissues. Glucose Ra and arterial plasma glucose concentration were similar after the two diets. After the High-CHO diet, arterial lactate concentration was higher and total carbohydrate oxidation rate well exceeded glucose Ra in plasma. Therefore, alterations in intracellular mechanisms may limit fatty acid oxidation after high-carbohydrate diets.
\end{abstract}

Diet: Fatty acids: Glucose: Oxidation: Stable isotopes

It is well established that carbohydrate and fat metabolism are closely interrelated (Randle et al. 1963; Sidossis \& Wolfe, 1996), although the mechanisms regulating their relative oxidation rates are not entirely understood. In man, the carbohydrate and fat contents of the diet have a major influence on substrate oxidation. Previous studies in healthy men have shown that post-absorptive fat oxidation is lower after a low-fat, high-carbohydrate diet than after an isoenergetic high-fat, low-carbohydrate diet (Bisschop et al. 2002). Suppressed post-absorptive fat oxidation is observed even when high-carbohydrate diets are compared with normal diets (Parks et al. 1999).

The mechanisms for the suppression of post-absorptive fat oxidation after high-carbohydrate diets are unclear. Since plasma non-esterified fatty acids (NEFA) are the major source for fat oxidation at rest (Sidossis \& Wolfe, 1996; Mensink et al. 2001), a possible mechanism is that low-fat, high-carbohydrate diets reduce NEFA availability (Helge et al. 2001; Bisschop et al. 2002), which in turn limits fat oxidation. Apart from a study that employed a very short-term (4 d) dietary intervention protocol (Schrauwen et al. 2000), there is no information on the effect of a longer-term highcarbohydrate diet on components of fatty acid metabolism in healthy human subjects, which would help to clarify the operation of such a mechanism.

Alterations in intracellular mechanisms could also lead to suppression of fat oxidation after high-carbohydrate diets. For example, it has been shown that short-term hyperglycaemia-hyperinsulinaemia decreases whole-body fat oxidation even in the setting of constant plasma NEFA availability (Sidossis \& Wolfe, 1996; Sidossis et al. 1996, 1999). This effect is primarily mediated via an increase in glycolytic flux, which blocks long-chain fatty acid entry into the mitochondria (Sidossis et al. 1996). It is possible that consumption of high-carbohydrate diets affect fat oxidation via a similar mechanism.

The purpose of the present study was to investigate the effect of isoenergetic low- and high-carbohydrate diets on various components of post-absorptive fatty acid metabolism, such as the rate of appearance (Ra) of NEFA, plasma-derived fatty acid oxidation and total fat oxidation, in healthy men. In addition, the effect of diet composition

\footnotetext{
Abbreviations: GCRC, General Clinical Research Center; High-CHO diet, high-carbohydrate diet; Low-CHO diet, low-carbohydrate diet; NEFA, non-esterified fatty acids; PDH, pyruvate dehydrogenase; Ra, rate of appearance.

* Corresponding author: Dr Labros Sidossis, fax +30 2109514759, email 1sidossis@hua.gr
} 
on aspects of post-absorptive glucose metabolism (glucose $\mathrm{Ra}$ and total carbohydrate oxidation) was assessed. This was accomplished by the use of stable-isotope tracer methodology and indirect calorimetry. Diets of 2-week duration based on real foods were employed. The results will potentially contribute to understanding the regulation of substrate oxidation by diet composition.

\section{Methods}

\section{Subjects}

Five men (age 35 (SD 4) years; body weight 73 (SD 3) kg; BMI 22 (SD 1) $\mathrm{kg} / \mathrm{m}^{2}$ ) participated in the study. They were all healthy, as indicated by comprehensive history and physical examination, and standard blood and urine tests. All subjects had stable body weights for at least 6 months before the study, normal oral glucose tolerance and did not exercise during the interventions. The study was approved by the Institutional Review Board and the General Clinical Research Center (GCRC) of the University of Texas Medical Branch at Galveston. Informed consent was obtained from all volunteers before enrolment.

\section{Experimental design}

Diet interventions. Detailed description of the diet interventions has been presented elsewhere (Mittendorfer \& Sidossis, 2001). In brief, subjects were admitted to the GCRC at the University of Texas Medical Branch on two different occasions. On one occasion, subjects received a low-carbohydrate (Low-CHO) diet (30\% energy intake from carbohydrates, $55 \%$ from fat, $15 \%$ from protein) for $14 \mathrm{~d}$; on the other occasion, subjects received a high-carbohydrate (High-CHO) diet (energy intake 75,10 and $15 \%$ from carbohydrates, fat and protein respectively) for $14 \mathrm{~d}$. In the Low-CHO diet, the contribution of sugars and starch to total energy intake was 12 and $18 \%$ respectively, and 35 and $40 \%$ in the High-CHO diet. Saturated and monounsaturated fatty acids represented 34 and $13 \%$ respectively of total energy intake in the Low-CHO diet and 7 and $1.4 \%$ in the High-CHO diet. Total daily energy supply was estimated to cover each subject's resting energy expenditure plus $20 \%$ to account for daily activities. All meals were prepared by the metabolic kitchen at the GCRC and consisted exclusively of regular food items (for example, pasta, meat, salads, fruit, cereal bars, dairy products). While in the GCRC, subjects were not allowed to leave for more than $15 \mathrm{~min}$ at a time so that their food intake could be monitored closely. The two interventions were randomised in order and performed 5 to 6 weeks apart. All subjects maintained their body weight during and between the interventions.

Isotope infusion. On the evening of the 14th day the volunteers ate dinner at 19.00 hours; afterwards they fasted until the completion of the tracer infusion (approximately $15 \mathrm{~h}$ later) the next day. At 20.30 hours Teflon catheters were placed percutaneously into an antecubital vein for isotope infusion and into a contralateral dorsal hand vein for blood sampling. The hand vein was heated during sampling in order to obtain arterialised blood samples. At 21.00 hours a bolus of $\mathrm{NaH}^{13} \mathrm{CO}_{3}$ $(25 \mu \mathrm{mol} / \mathrm{kg})$ was given and a continuous infusion $\left(0.035 \mu \mathrm{mol} / \mathrm{kg}\right.$ per min) of a $\left[\mathrm{U}_{-}{ }^{13} \mathrm{C}\right]$ fatty acid mixture (86\% enriched) bound to human albumin was started and maintained until the completion of the study (the next day). The long duration of infusion of the fatty acid mixture was necessary for the estimation of other metabolic parameters presented elsewhere (Mittendorfer \& Sidossis, 2001). At 07.00 hours the next day, the subjects were transferred to the Angiography and Interventional Radiology Center of the University of Texas Medical Branch, where a catheter was inserted into the femoral artery, as described previously (Sidossis et al. 1999). After catheter placement, the subjects were transferred back to the GCRC for completion of the study. The $\left[\mathrm{U}_{-}{ }^{13} \mathrm{C}\right]$ fatty acid infusion continued uninterrupted during the catheter placement by use of a battery-operated pump. At 60 to $90 \mathrm{~min}$ after catheter placement, a primed $(18 \mu \mathrm{mol} / \mathrm{kg})$ continuous $(0.22 \mu \mathrm{mol} / \mathrm{kg}$ per min $)$ infusion of $\left[6,6-{ }^{2} \mathrm{H}_{2}\right]$ glucose was started and maintained for $120 \mathrm{~min}$. Calibrated syringe pumps (Harvard Apparatus, South Natick, MA, USA) were used for all infusions. For each infusion study, samples of the isotope infusates were analysed for the exact enrichment and concentration of tracers. All tracer infusions were stopped after the last blood sample was obtained and the catheters were removed immediately.

Blood and breath sampling. For the determination of background plasma NEFA and glucose enrichment and background breath $\mathrm{CO}_{2}$ enrichment, blood and breath samples were obtained in the evening of the 14th day before the beginning of the tracer infusion. For the determination of plasma insulin, NEFA and glucose concentrations and plasma NEFA and glucose enrichment, blood samples were obtained from the femoral artery every $10 \mathrm{~min}$ during the last $30 \mathrm{~min}$ of the tracer infusion. Blood samples for the determination of NEFA and glucose concentration and enrichment and for the determination of insulin concentration were collected into pre-chilled tubes containing ethylene glycol-bis( $\beta$-aminoethylether)$\mathrm{N}, \mathrm{N}, \mathrm{N}^{\prime}, \mathrm{N}^{\prime}$-tetra-acetic acid as anticoagulant. This procedure has been shown to prevent ex vivo lipolysis of triacylglycerol in plasma from subjects that were given heparin (Hargreaves et al. 1991). Blood samples for the determination of glucose kinetics were collected into pre-chilled tubes containing fluoride. Plasma from all samples was separated immediately after the completion of the study and stored at $-20^{\circ} \mathrm{C}$ until analysis.

Whole-body $\mathrm{O}_{2}$ consumption $\left(\mathrm{V}_{2}\right)$ and $\mathrm{CO}_{2}$ production $\left(\mathrm{VCO}_{2}\right)$ were measured over 30 min during the last hour of the study using a metabolic cart (Sensormedics 2900; Yorba Linda, CA, USA). For the determination of ${ }^{13} \mathrm{CO}_{2}$ enrichment in expired air, four breath samples were collected into $20 \mathrm{ml}$ vacutainers every $10 \mathrm{~min}$ during the last 30 min of the tracer infusion.

\section{Materials}

All isotopes were purchased from Cambridge Isotope Laboratories (Andover, MA, USA). The composition of 
the $\left[\mathrm{U}-{ }^{13} \mathrm{C}\right]$ fatty acid mixture was: $51.7 \%$ palmitic acid (C16:0), 9.3\% palmitoleic acid (C16:1), 5.5\% stearic acid (C18:0), 17.9\% oleic acid (C18:1), 14.1\% linoleic acid $(\mathrm{C} 18: 2)$ and $1.5 \%$ linolenic acid $(\mathrm{C} 18: 3)$. Human albumin $(5 \%$, w/v) was purchased from Baxter Healthcare Corporation (Glendale, CA, USA).

\section{Analytical procedures}

The ${ }^{13} \mathrm{CO}_{2}:{ }^{12} \mathrm{CO}_{2}$ value in expired air samples was determined by isotope ratio MS (SIRA VG Isotech, Cheshire, UK) as previously described (Wolfe, 1992; Sidossis et al. 1999). For the determination of plasma NEFA enrichments and concentrations, fatty acids were extracted from plasma and isolated by TLC (Wolfe, 1992). After extraction from the TLC silica gel, fatty acids were combusted (Elemental Analyzer, Carlo Erba 1500; CE Elantech, Inc., Lakewood, $\mathrm{NJ}$, USA), and the ${ }^{13} \mathrm{CO}_{2} \cdot{ }^{12} \mathrm{CO}_{2}$ value in the headspace was determined by isotope ratio MS. Plasma glucose enrichment was determined after derivatisation with penta-acetate (Wolfe, 1992). Plasma glucose, lactate, 3-hydroxybutyrate and insulin concentrations were measured as previously described (Mittendorfer \& Sidossis, 2001).

\section{Calculations}

The rates of whole-body fat and carbohydrate oxidation were calculated by indirect calorimetry based on the $\mathrm{O}_{2}$ consumption and $\mathrm{CO}_{2}$ production rates (Frayn, 1983). Total fatty acid oxidation was calculated by converting the rate of fat (triacylglycerol) oxidation $(\mathrm{g} / \mathrm{kg}$ per $\mathrm{min}$ ) to its molar equivalent, assuming the average molecular weight of triacylglycerol to be $860 \mathrm{~g} / \mathrm{mol}$ and multiplying the molar rate of triacylglycerol oxidation by three, because each molecule contains three moles of fatty acids.

NEFA and glucose kinetics were calculated using the average femoral arterial NEFA, glucose and $\mathrm{CO}_{2}$ enrichments and concentrations obtained during the last $30 \mathrm{~min}$ of the tracer infusion. An isotopic plateau for plasma NEFA and glucose carbon enrichment, and blood and breath $\mathrm{CO}_{2}$ carbon enrichment was reached when the measurements for the calculation of fatty acid and glucose kinetics were made. This protocol has been described previously (Sidossis et al. 1999).

The $\mathrm{Ra}$ (which was equal to the rate of disappearance) of NEFA and glucose in plasma was determined by dividing the tracer infusion rate $(\mathrm{I})$ by the arterial enrichment $\left(\mathrm{E}_{\mathrm{A}}\right)$ of NEFA and glucose respectively, i.e.

$$
\mathrm{Ra}=\mathrm{I} / \mathrm{E}_{\mathrm{A}} .
$$

Plasma NEFA oxidation was calculated from the tracer data as follows:

$$
\text { Plasma NEFA oxidation }=\frac{\mathrm{E}_{\mathrm{CO} 2} \times \dot{\mathrm{VCO}}_{2}}{\mathrm{E}_{\mathrm{A}} \times \mathrm{ACF} \times 17},
$$

where $\mathrm{E}_{\mathrm{CO} 2}$ is breath ${ }^{13} \mathrm{CO}_{2}$ enrichment, $\mathrm{ACF}$ is the acetate correction factor obtained after $15 \mathrm{~h}$ of labelled acetate infusion (Mittendorfer et al. 1998) to account for carbonlabel fixation that occurs during oxidation of fatty acids (Sidossis et al. 1995) and 17 is the mean number of ${ }^{13} \mathrm{C}$ atoms in our NEFA mixture.

\section{Statistical analysis}

All data are reported as mean values with their standard errors. The effect of diets on substrate concentrations, kinetics and oxidation was evaluated using paired Student's $t$ tests.

\section{Results}

Arterial concentrations of plasma metabolites during the last 30 minutes of the tracer infusion

The average arterial plasma NEFA, glucose, lactate, insulin and 3-hydroxybutyrate concentrations are presented in Table 1. Plasma NEFA concentration was $35 \%$ lower $(P=0.048)$ after the High-CHO than after the Low-CHO diet. Glucose and insulin concentrations were similar following the two diets. Lactate concentration was $50 \%$ higher $(P=0.024)$ after the High-CHO diet than after the Low-CHO diet. Plasma 3-hydroxybutyrate concentration tended to be lower after the High-CHO diet $(P=0.07)$ (Table 1).

\section{Substrate kinetics}

Plasma NEFA Ra was about $35 \%$ lower $(P=0.045)$ following the High-CHO than the Low-CHO diet (Fig. 1). Plasma glucose $\mathrm{Ra}$ was similar following the Low-CHO and the High-CHO diet (Fig. 1).

Table 1. Post-absorptive (after $15 \mathrm{~h}$ fast) concentrations of plasma metabolites in the femoral artery after the low- and high-carbohydrate $(\mathrm{CHO})$ diet $†$

\begin{tabular}{|c|c|c|c|c|}
\hline & \multicolumn{2}{|c|}{ Low-CHO diet } & \multicolumn{2}{|c|}{ High-CHO diet } \\
\hline & Mean & SE & Mean & SE \\
\hline Non-esterified fatty acids $(\mu \mathrm{mol} / \mathrm{l})$ & 299 & 35 & $190^{*}$ & 33 \\
\hline Glucose $(\mathrm{mmol} / \mathrm{l})$ & 4.9 & 0.1 & 4.9 & 0.1 \\
\hline Insulin (pmol/l) & 34 & 10 & 46 & 12 \\
\hline Lactate (mmol//) & 0.64 & 0.07 & $0.98^{\star *}$ & 0.08 \\
\hline 3-Hydroxybutyrate $(\mathrm{mmol} / \mathrm{l})$ & 0.21 & 0.03 & 0.15 & 0.04 \\
\hline
\end{tabular}

(Mean values with their standard errors for five men)

Mean values were significantly different from those for the Low-CHO diet: ${ }^{*} P=0.048,{ }^{\star *} P=0.024$. †For details of diets and procedures, see p. 414. 


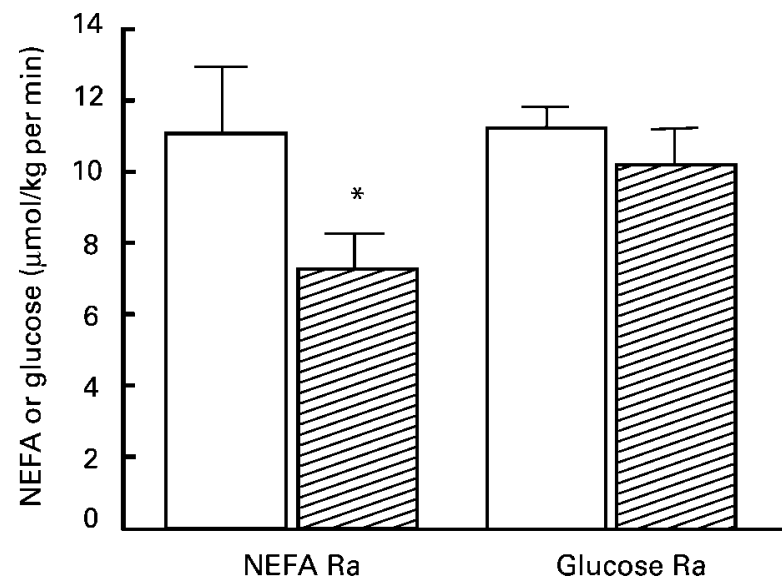

Fig. 1. Rate of appearance (Ra) of non-esterified fatty acids (NEFA) and glucose following the low-carbohydrate ( $\square$ ) and the high-carbohydrate $(\mathbb{Q})$ diet. Values are means for five men with standard errors shown by vertical bars. *Mean value was significantly lower than that for the low-carbohydrate diet $(P=0.045)$.

\section{Substrate oxidation}

The rates of total fatty acid oxidation (indirect calorimetry) and plasma fatty acid oxidation (tracer method) were both significantly lower following the High-CHO than the Low-CHO diet (Table 2). On the contrary, carbohydrate oxidation (indirect calorimetry) was higher $(P<0 \cdot 001)$ after the High-CHO diet (Table 2). As a result, the relative contribution of carbohydrates to the total energy expenditure was higher $(P=0.006)$ after the High-CHO diet, whereas fat oxidation was higher $(P=0.006)$ after the Low-CHO diet (Fig. 2). Total energy expenditure did not differ between the two diets.

\section{Discussion}

To our knowledge, the present study is the first to describe comprehensively the effect of isoenergetic low- and highcarbohydrate diets on post-absorptive substrate kinetics and oxidation in healthy men. The in-patient setting of the study ensured high compliance to the diets, which comprised real foods. It was found that NEFA release (NEFA $\mathrm{Ra}$ ), plasma and total fatty acid oxidation were all suppressed after 2 weeks on the High-CHO diet as compared with 2 weeks on the Low-CHO diet.

A lower NEFA Ra after the High-CHO than the Low$\mathrm{CHO}$ diet suggests a decrease in peripheral lipolysis after the High-CHO diet and/or increased fatty acid re-esterification within adipose tissue. Similar responses in NEFA Ra have been observed previously after liquid low- and high-carbohydrate diets in healthy men (Bisschop et al. 2002). Several factors control the release of fatty acids from adipose tissue. Only insulin was measured and it is known that lipolysis is very sensitive to even minimal changes in insulin concentration (Nurjhan et al. 1986; Jensen et al. 1989). The small, although statistically insignificant, increase in basal insulin concentration after the High-CHO diet may have contributed to the observed decrease in NEFA Ra. Furthermore, a previous report (Bisschop et al. 2001) showed that, during a hyperinsulinaemic-euglycaemic clamp, NEFA concentrations were higher after a low- than the high-carbohydrate diet, indicating reduced insulin sensitivity with regard to the effect of insulin on lipolysis after the low-carbohydrate diet.

An effect of the carbohydrate and fat content of the diets on post-absorptive endogenous glucose production was not observed, although the methods that were employed cannot distinguish between hepatic glycogenolysis and gluconeogenesis. Our finding is in contrast with the results of a previous study (Bisschop et al. 2000), which found higher glucose $\mathrm{Ra}$ after a high-carbohydrate than after an isoenergetic low-carbohydrate diet. This discrepancy may be due to differences in study design and specifically the composition and physical state of the diets used. In the study by Bisschop et al. (2000), the high-carbohydrate diet was a fat-free liquid formula and the low-carbohydrate diet was a liquid formula containing only $2 \%$ energy in the form of carbohydrate. Furthermore, the physical state of the meal (liquid $v$. solid) affects hormonal and metabolic responses (Habas \& Macdonald, 1998). Therefore, dietary interventions using liquid diets may exert different metabolic effects compared with real-food diets.

After both the low- and the high-carbohydrate diet plasma-derived fatty acids constituted the major source for fat oxidation (Table 2). Thus, the decrease in plasma fatty acid oxidation after the High-CHO diet was reflected on total fatty acid oxidation. In agreement with other studies (Helge et al. 2001; Bisschop et al. 2002), it was found that the average arterial plasma NEFA concentration was lower after the consumption of the High-CHO diet, and this could have theoretically limited fat oxidation. However, even though reduced, NEFA Ra after the High$\mathrm{CHO}$ diet $(7.2$ (SE 1.0$) \mu \mathrm{mol} / \mathrm{kg}$ per $\min$ ) was more than threefold higher than plasma fatty acid oxidation

Table 2. Substrate oxidation rates after the low- and high-carbohydrate $(\mathrm{CHO})$ diet† (Mean values with their standard errors for five men)

\begin{tabular}{|c|c|c|c|c|}
\hline & \multicolumn{2}{|c|}{ Low-CHO diet } & \multicolumn{2}{|c|}{ High-CHO diet } \\
\hline & Mean & SE & Mean & SE \\
\hline Total fatty acid oxidation ( $\mu \mathrm{mol} / \mathrm{kg}$ per $\min )$ & $4 \cdot 8$ & 0.5 & $2 \cdot 4^{\star}$ & 0.7 \\
\hline Plasma fatty acid oxidation ( $\mu \mathrm{mol} / \mathrm{kg}$ per min) & $4 \cdot 6$ & 0.8 & $2 \cdot 1^{\star \star}$ & 0.4 \\
\hline Carbohydrate oxidation ( $\mu \mathrm{mol} / \mathrm{kg}$ per $\min$ ) & $10 \cdot 7$ & $2 \cdot 4$ & $19 \cdot 7^{\star \star \star}$ & $1 \cdot 0$ \\
\hline
\end{tabular}

Mean values were significantly different from those for the Low-CHO diet: * $P=0.027$, ** $P=0.041$, *** $P<0.001$.

† For details of diets and procedures, see p. 414. 


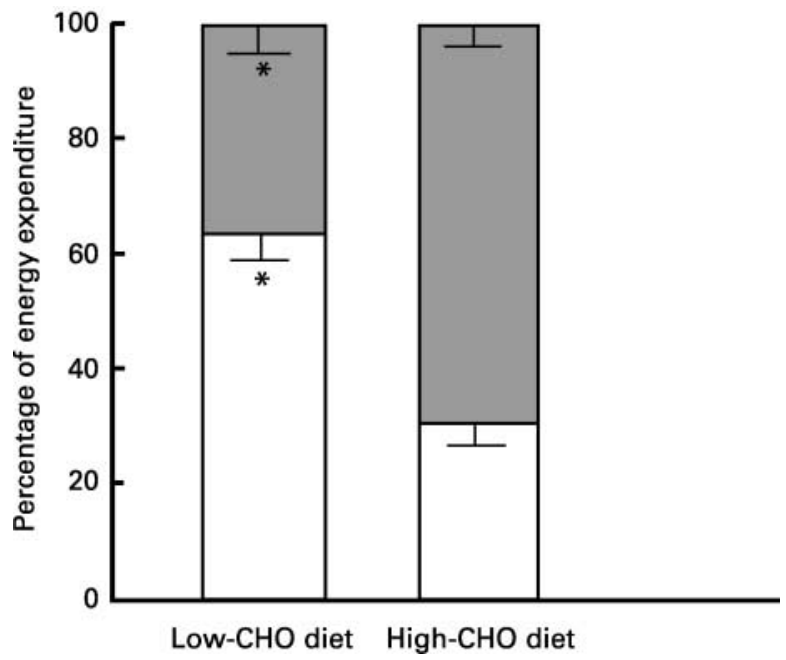

Fig. 2. Relative contribution of fat $(\square)$ and carbohydrates $(\square)$ to total energy expenditure at the whole-body level (indirect calorimetry) following the low- and the high-carbohydrate $(\mathrm{CHO})$ diets. Values are means for five men with standard errors shown by vertical bars. *Mean values were significantly different from those for the high-carbohydrate diet $(P=0.006)$.

(2.1 (SE 0.4) $\mu \mathrm{mol} / \mathrm{kg}$ per min) arguing against the possibility that NEFA availability was a limiting factor for fatty acid oxidation.

Alternatively, changes in intracellular mechanisms may have inhibited fatty acid oxidation after the High-CHO diet. It has been shown that glucose availability regulates whole-body fatty acid oxidation (Sidossis \& Wolfe, 1996; Sidossis et al. 1996, 1999). This is mediated via an increase in glycolytic flux which, in turn, blocks longchain fatty acid entry into the mitochondria (Sidossis et al. 1996). In the present study, systemic plasma glucose concentrations and glucose $\mathrm{Ra}$ were similar following the Low- and the High-CHO diet (Table 1, Fig. 1). This finding, at first, argues against a high glycolytic rate-induced inhibition of fat oxidation as a possible mechanism to explain the observed decrease in fat oxidation after the High-CHO diet. It is possible, however, that glycogenolysis and thus glycolytic flux was higher following the High-CHO diet compared with the Low-CHO diet. This is supported by the observation that following the HighCHO diet, carbohydrate oxidation (19.7 (SE 1.0) $\mu \mathrm{mol} / \mathrm{kg}$ per min) well exceeded glucose $\mathrm{Ra}$ in plasma $(10 \cdot 2$ (SE 0.9) $\mu \mathrm{mol} / \mathrm{kg}$ per min) whereas, after the Low-CHO diet, the rate of whole-body carbohydrate oxidation (10.7 (SE 2.4) $\mu \mathrm{mol} / \mathrm{kg}$ per min) was fully accounted for by the glucose $\mathrm{Ra}$ in plasma (11.2 (SE 0.6) $\mu \mathrm{mol} / \mathrm{kg}$ per min). This suggests that glycogen stores were utilised to a greater extent following the High-CHO diet. Also, the higher lactate concentration after the High- $\mathrm{CHO}$ diet suggests higher glycolytic flux after this diet.

In the present study, a control diet was not included and so it cannot be determined to what extent the Low$\mathrm{CHO}$ diet shifted fuel reliance to fat to spare carbohydrate. Low-CHO diets have been shown to increase resting human skeletal muscle pyruvate dehydrogenase (PDH) kinase activity and decrease the amount of $\mathrm{PDH}$ in its active form (Peters et al. 1998), which in turn decreases carbohydrate oxidation. Therefore, effects of the Low$\mathrm{CHO}$ diet on PDH activity may have also influenced carbohydrate and fat oxidation rates in the present study.

In summary, it has been shown that, in healthy men, consumption of an isoenergetic, High-CHO diet for 2 weeks increases post-absorptive carbohydrate oxidation and decreases total and plasma fatty acid oxidation as compared with a Low-CHO diet. The observed suppression of fat oxidation after the High-CHO diet is unlikely to have resulted from decreased fatty acid delivery to the tissues. A potential mechanism for the High- $\mathrm{CHO}$ diet-induced reduction in fatty acid oxidation is a decrease in the proportion of NEFA uptake that is channelled into oxidative pathways due to glucose-induced inhibition of long-chain fatty acid entry into the mitochondria. In addition, the Low-CHO diet may have affected carbohydrate and fat oxidation rates via effects on PDH activity.

\section{Acknowledgements}

This work was supported by National Institutes of Health Grants DK-51969 and MO1-00073 (Mass Spec Resource), American Heart Association Texas Affiliate Grant 96G1618, a Fellowship from the European Society of Parenteral and Enteral Nutrition, and Shriners' Hospital Grant 15849. The authors thank the nursing and dietary staff of the General Clinical Research Center and the Department of Radiology for their help in the performance of the experiments.

\section{References}

Bisschop PH, Ackermans MT, Endert E, et al. (2002) The effect of carbohydrate and fat variation in euenergetic diets on postabsorptive free fatty acid release. Br J Nutr 87, 555-559.

Bisschop PH, de Metz J, Ackermans MT, et al. (2001) Dietary fat content alters insulin-mediated glucose metabolism in healthy men. Am J Clin Nutr 73, 554-559.

Bisschop PH, Pereira Arias AM, Ackermans MT, et al. (2000) The effects of carbohydrate variation in isocaloric diets on glycogenolysis and gluconeogenesis in healthy men. J Clin Endocrinol Metab 85, 1963-1967.

Frayn KN (1983) Calculation of substrate oxidation rates in vivo from gaseous exchange. J Appl Physiol 55, 628-634.

Habas ME \& Macdonald IA (1998) Metabolic and cardiovascular responses to liquid and solid test meals. Br J Nutr 79, 241-247.

Hargreaves M, Kiens B \& Richter EA (1991) Effect of increased plasma free fatty acid concentrations on muscle metabolism in exercising men. J Appl Physiol 70, 194-201.

Helge JW, Watt PW, Richter EA, Rennie MJ \& Kiens B (2001) Fat utilization during exercise: adaptation to a fat-rich diet increases utilization of plasma fatty acids and very low density lipoprotein-triacylglycerol in humans. $J$ Physiol 537, $1009-1020$.

Jensen MD, Caruso M, Heiling V \& Miles JM (1989) Insulin regulation of lipolysis in nondiabetic and IDDM subjects. Diabetes 38, 1595-1601.

Mensink M, Blaak EE, van Baak MA, Wagenmakers AJM \& Saris WHM (2001) Plasma free fatty acid uptake and oxidation are already diminished in subjects at high-risk for developing type 2 diabetes. Diabetes 50, 2548-2554.

Mittendorfer B \& Sidossis LS (2001) Mechanism for the increase in plasma triacylglycerol concentrations after consumption of 
short-term, high-carbohydrate diets. Am J Clin Nutr 73, 892-899.

Mittendorfer B, Sidossis LS, Walser E, Chinkes D \& Wolfe RR (1998) Regional acetate kinetics and oxidation in human volunteers. Am J Physiol 274, E978-E983.

Nurjhan N, Campbell PJ, Kennedy FP, Miles JM \& Gerich JE (1986) Insulin dose-response characteristics for suppression of glycerol release and conversion to glucose in humans. Diabetes 35, 1326-1331.

Parks EJ, Krauss RM, Christiansen MP, Neese RA \& Hellerstein MK (1999) Effects of low-fat, high-carbohydrate diet on VLDL-triglyceride assembly, production, and clearance. J Clin Invest 104, 1087-1096.

Peters SJ, Amand TAS, Howlett RA, Heigenhauser GJF \& Spriet LL (1998) Human skeletal muscle pyruvate dehydrogenase kinase activity increases after a low-carbohydrate diet. Am J Physiol 275, E980-E986.

Randle PJ, Garland PB, Hales CN \& Newsholme EA (1963) The glucose-fatty acid cycle. Its role in insulin sensitivity and the metabolic disturbances of diabetes mellitus. Lancet $\mathbf{i}$, $785-789$.
Schrauwen P, Wagenmakers AJM, van Marken Lichtenbelt WD, Saris WHM \& Westerterp KR (2000) Increase in fat oxidation on a high-fat diet is accompanied by an increase in triglyceridederived fatty-acid oxidation. Diabetes 49, 640-646.

Sidossis LS, Coggan AR, Gastaldelli A \& Wolfe RR (1995) A new correction factor for use in tracer estimations of plasma fatty acid oxidation. Am J Physiol 269, E649-E656.

Sidossis LS, Mittendorfer B, Chinkes D, Walser E \& Wolfe RR (1999) Effect of hyperglycemia-hyperinsulinemia on whole body and regional fatty acid metabolism. Am J Physiol 276, E427-E434.

Sidossis LS, Stuart CA, Schulman GI, Lopaschuk GD \& Wolfe RR (1996) Glucose plus insulin regulate fat oxidation by controlling the rate of fatty acid entry into the mitochondria. $J$ Clin Invest 98, 2244-2250.

Sidossis LS \& Wolfe RR (1996) Glucose and insulin-induced inhibition of fatty acid oxidation: the glucose-fatty acid cycle reversed. Am J Physiol 270, E733-E738.

Wolfe RR (1992) Radioactive and Stable Isotope Tracers in Biomedicine: Principles and Practice of Kinetic Analysis. New York, NY: Wiley-Liss. 